\title{
7
}

\section{Spectrophotometric Methods as Solutions to Pharmaceutical Analysis of $\beta$-Lactam Antibiotics}

\author{
Judyta Cielecka-Piontek ${ }^{1}$, Przemysław Zalewski \\ Anna Krause ${ }^{2}$ and Marek Milewski ${ }^{2}$ \\ ${ }^{1}$ Poznan University of Medical Sciences, Department of Pharmaceutical Chemistry \\ ${ }^{2}$ PozLab Contract Research Organization at Centre of Transfer of Medical Technologies
}

Poland

\section{Introduction}

Following the discovery of the first analog of penicillin by A. Fleming (1929), the $\beta$-lactam antibiotics are still a developing group of chemotherapeutics and are used in treatment of majority of diseases with bacterial etiology. $\beta$-lactam antibiotics have a broad spectrum of antibacterial activity, favourable pharmacokinetic parameters and low side effects. In $\beta$ lactam therapy two main problems are still current. The increasing resistance of some bacterial strains which implicates necessity to combine the therapy with inhibitors of $\beta$ lactamases and other chemotherapeutics. The second problem of therapy of $\beta$-lactam antibiotics is their significant instability [1-3]. The analogs from that group are easily degraded in aqueous solutions and in solid state. They are a special group of drugs because parallel to losing the antibacterial efficiency, the strong allergic properties can also appear as a results of their degradation. Therefore in terms of quality control, the stability of $\beta$-lactam antibiotics in solutions was widely studied. The evaluation of stability concerned also the studies of their metabolites and intravenous solutions after preparations of pharmaceutical dosage forms. Moreover, the evaluation of concentration changes during storage of substance in solid state was also conducted. As problem of the instability of some $\beta$-lactam analogs has been solved their oral administration is possible. An intake of oral formulations is connected with appearance of excipients, which can influence rate of degradation and cause formation of different degradation products.

The common element of chemical structure of all $\beta$-lactam antibiotics is five-membered $\beta$ lactam ring. Currently, higher significance in treatment have derivatives in which the $\beta$ lactam ring is fused to:

- thiazolidine ring in penam analogs,

- 2,3-dihydro-2H-1,3-tiazine ring in cephem analogs,

- 2,3-dihydro-1H-pyrrole in carbapenem analogs,

- 2,3-dihydrotiazole in penem analogs (Fig .1).

These connections implicate the different intra-ring stress. The presence of sulphur atom and/or double bonds influence on length of bond and intra-molecular angle in molecule of $\beta$-lactam analog. Finally for some derivatives, the differences in stability are noticeable. Additionally, the factor distinguishing a stability of derivatives of $\beta$-lactam analogs are 


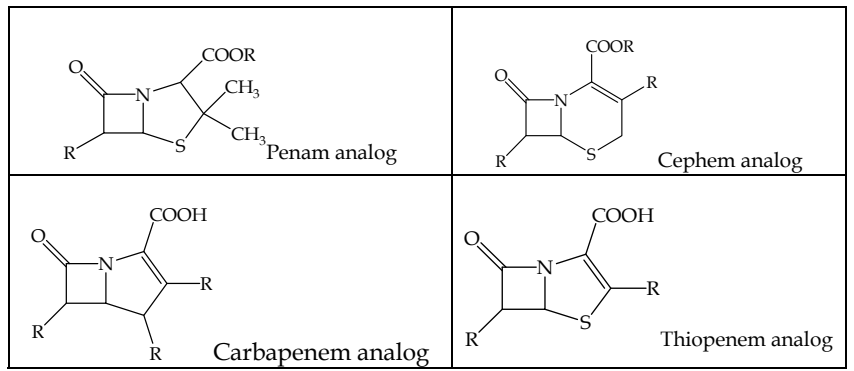

Fig. 1. Chemical structure of penam, cephem, carbapenem and thiopenem nuclei.

chemical structures of substituents at C2, C3, C5, C6, and C7. The amount and type of degradation products of $\beta$-lactam antibiotics often depend on affecting factors (solvents, concentration of substance and hydrogen ions, temperature). Moreover, most of the $\beta$ lactam antibiotics obtained by chemical synthesis or fermentation contain impurities being remnants of the process. In the development of analytical methods for the determination of $\beta$-lactam antibiotics, selectivity is a fundamental validation parameter. A reliable, selective method is expected to allow separation and determination of parental substance in the presence of related ones. Current International Conference on Harmonization (ICH) guidelines require the development of analytical methods permitting analysis in the presence of related products (Q1A-R2) [4]. These requirements are restrictions but also challenges during the development of analytical methods for the determination of $\beta$-lactam antibiotics. The problem of the overlapping of the "background" originating from related products (impurities, degradation products and metabolites) and/or the presence of other active substances in a sample (inhibitors of $\beta$-lactamases, other drugs) was solved during the determination of $\beta$-lactam antibiotics by using chromatographic techniques (highperformance liquid chromatography, thin layer chromatography). On the other hand, search of new solutions and analytical methods, especially being in accordance with the "green chemistry" concept, is very important and up-to-date. Analytical methods based on determination of spectrophotometric properties of $\beta$-lactam analogs are a developing tools in their analysis. Non-destructive investigations of $\beta$-lactam analogs, did not producing residues, were reported in fields of many spectrophotometric methods. A few methods of determination of $\beta$-lactam analogs were developed by using infrared spectrophotometry enriched by chemometric procedures [5-6]. Most of all analytical methods for the determination of $\beta$-lactam analogs were developed in range of visible and ultraviolet radiations. Desired, selective signals were possible to obtain by application of following techniques:

- $\quad$ as spectrophotometric methods

- direct spectrophotometry

- direct spectrophotometry enriched by chemometric procedures

- derivative spetrophotometry

- derivative spectrotometry enriched by chemometric procedures

- $\quad$ as visible spectrophotometric methods

- measurement of absorption of species being a result of reaction between analyte and derivatizating reagent

- measurement of absorption of species being a result of reaction between degradation products of analyte and derivatizating reagent (Table 1). 


\begin{tabular}{|c|c|c|c|c|c|}
\hline \multirow[t]{2}{*}{ Derivative } & \multicolumn{3}{|c|}{ Ultraviolet region } & \multicolumn{2}{|c|}{ Visible region } \\
\hline & 1 & 2 & 3 & $1^{*}$ & $2^{*}$ \\
\hline Analog of penam & $\checkmark$ & $\checkmark$ & $\checkmark$ & $\checkmark$ & $\checkmark$ \\
\hline \multicolumn{6}{|l|}{ Analog of cephem } \\
\hline I generation & $\checkmark$ & $\checkmark$ & $\checkmark$ & $\checkmark$ & $\checkmark$ \\
\hline II generation & & $\checkmark$ & & $\checkmark$ & $\checkmark$ \\
\hline III generation & $\checkmark$ & $\checkmark$ & $\checkmark$ & $\checkmark$ & $\checkmark$ \\
\hline IV generation & & $\checkmark$ & & & \\
\hline Analog of carbapenem & $\checkmark$ & $\checkmark$ & $\checkmark$ & & \\
\hline Analog of penam & & $\checkmark$ & & & \\
\hline \multicolumn{6}{|c|}{$\begin{array}{l}\text { 1. direct spectrophotometry enriched by chemometric procedures } \\
\text { 2. derivative spetrophotometry } \\
\text { 3. derivative spectrotometry enriched by chemometric procedures } \\
\text { 1.* measurement of absorption of species being a result of reaction between analyte and } \\
\text { derivatizating reagent } \\
\text { 2.* measurement of absorption of species being a result of reaction between degradation } \\
\text { products of analyte and derivatizating reagent }\end{array}$} \\
\hline
\end{tabular}

Table 1. Possibilities of application of visible and ultraviolet spectrophotometric determinations for analysis of $\beta$-lactam antibiotics in the period of time 1994-2011.

\section{Spectrophotometric methods for determination of $\beta$-lactam antibiotics}

\subsection{Direct spectrophotometry}

Spectra of $\beta$-lactam antibiotics recorded by using direct spectrophotometry do not have desired selectivity due to the presence of related products. A comparison of sharp zeroorder spectra and/or value of absorption maxima for some $\beta$-lactam analogs with ones obtained for CRS (chemical reference substance) is recommended by pharmacopeias for an their identification [7] . Lack of desired absorbing species in chemical structure of penam analog often do not allow to apply direct spectrophotometry even for qualitative studies of substance of high purity.

Paradoxically, the significant instability of analogs can sometimes solve this problem due to formation of degradation products that can absorb ultraviolet radiation permitting determination of parental substance.

Significant susceptibility of $\beta$-lactam analogs to degradation in basic medium was reported during analysis of cephem analogs. It was confirmed that formation of piperazine-2,5-dione 
derivative, peak at $340 \mathrm{~nm}$, was possible via intra-molecular nucleophilic attack of the primary amine from the side chain on $\beta$-lactam ring ( $\mathrm{pH}=11$ was required) (Fig. 2) [8].

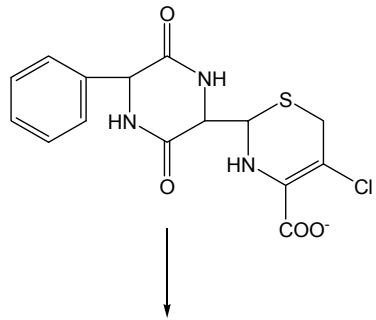

products

The degradation products of cefactor - piperazine2,5-dione derivative.

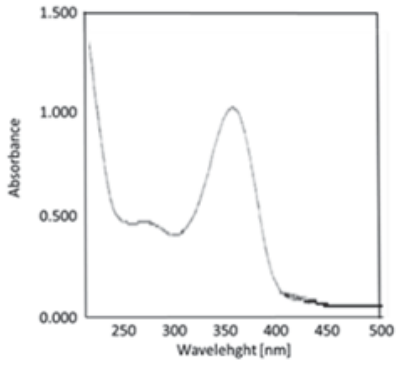

The spectrum of piperazine-2,5-dione derivative of cefaclor.

Fig. 2. Chemical structures of degradation products of cefaclor $(1.0 \mathrm{mmol} / \mathrm{l})$ formed at $\mathrm{pH}$ 11.0 and its spectrum [8].

The degradation of penam analogs in acidic conditions was also a base for spectrophotometric determination. As it is shown in Fig. 3, different pathways of degradation (including enzymatic one) can lead to obtaining absorbing species in the range of ultraviolet radiation. As a results of chemical degradation of penam analog in acidic conditions, the penicilloic acid, penillic acid and penicillenic acid are formed and absorb the ultraviolet radiation in the range $320-360 \mathrm{~nm}$, respectively [9]. While during the enzymatic degradation under the influence of penicillin acylase, D-4-hydroxyphenylglycine (D-HPhG) and 6-aminopenicillanic acid are formed. Then the D-HPhG was catalyzed by Dphenylglycine aminotransferase to form L-glutamate and hydroxybenzoylformate which strongly absorb UV light at $335 \mathrm{~nm}$ [10].

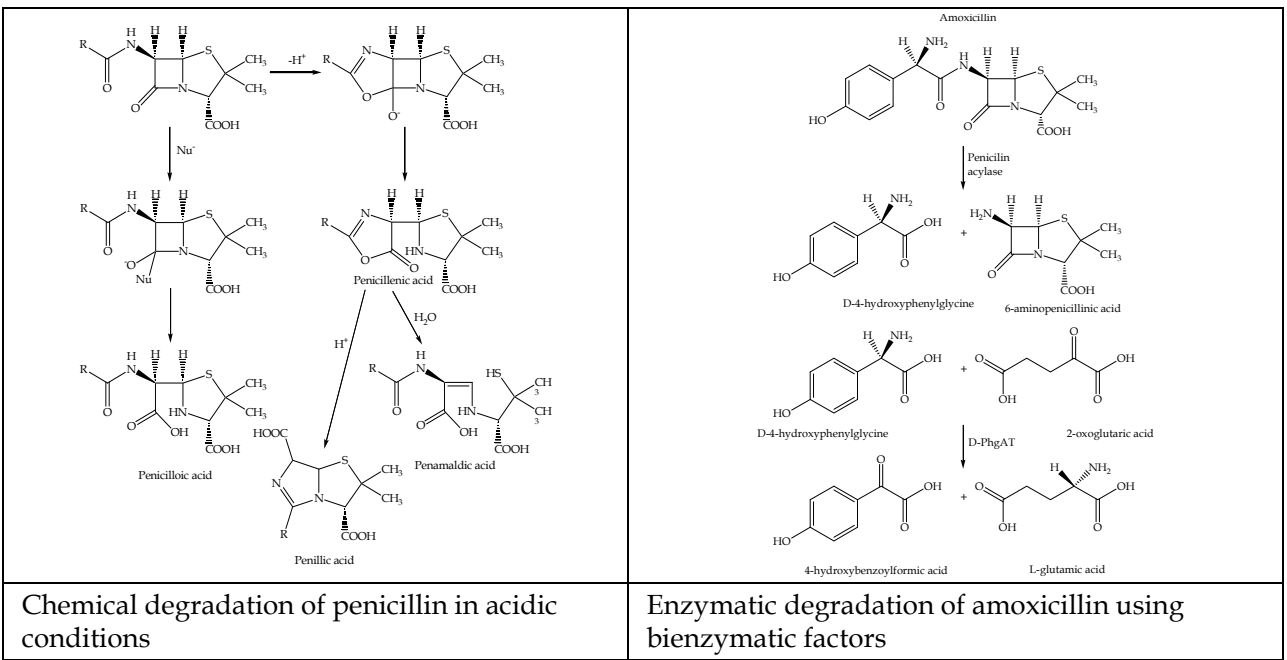

Fig. 3. The pathways of obtaining of absorbing degradation products of penam analog [9-10]. 


\subsection{Direct spectrophotometry enriched by chemometric procedures}

The other way of improving the selectivity of direct spectrophotometry for the determination of $\beta$-lactam antibiotics is the enrichment of data analysis by chemometric procedures. A literature review revealed the application of the following determinations of $\beta$-lactam antibiotics enriched by chemometric procedures that solved the problem of spectral overlap without additional separation techniques at the stage of sample preparation, were used:

- a separation of analog of cephem in the presence of impurities originating from synthesis (e.g., cephalexin in the presence of 7-aminocephalosporanic acid and acid-induced degradation products) using H-point standard additions method (HPSAM) [11]

- determination of analog of penam in the presence of other drugs (e.g., amoxicillin in the presence of diclofenac) using partial least squares (PLS) regression analysis [12]

- determination of analog of cephem in the presence of alkali-induced degradation products using full spectrum quantitation (FSQ) (e.g., cefotaxime, ceftazidine, ceftiaxome, in the presence of degradation products) [13].

Each chemometric method relies on different tools of regression analysis of multicomponent system permitting simultaneous determination of two or more components.

The determination of $\beta$-lactam analyte in the presence of known and unknown inferences was possible by the application of HPSAM procedure, where analyte concentration is calculated from the following equation:

$$
\frac{\left(\mathrm{A}_{0}-\mathrm{b}_{0}\right)+\left(\mathrm{A}^{\prime}-\mathrm{b}\right)}{\mathrm{M}\left(\lambda_{1}\right)-\mathrm{M}\left(\lambda_{2}\right)}=-\mathrm{C}_{\mathrm{X}}+\frac{\left(\mathrm{A}^{\prime}-\mathrm{b}\right)}{\mathrm{M}\left(\lambda_{1}\right)-\mathrm{M}\left(\lambda_{2}\right)}
$$

where $b_{0}$ and $A_{0}$ are the absorbance values for $\beta$-lactam analyte, $b$ and $A^{\prime}$ ones for the interferent, at $\lambda_{1}$ and $\lambda_{2}$ and $M\left(\lambda_{1}\right), M\left(\lambda_{2}\right)$ are slopes of plots at selected wavelengths.

In PLS technique, analytical sensibility was defined as $\gamma=\frac{\operatorname{SEN}_{\mathrm{k}}}{\left\|\sigma_{\mathrm{r}}\right\|}$ where $\mathrm{SEN}_{\mathrm{k}}=\frac{1}{\left\|\mathrm{~b}_{\mathrm{k}}\right\|}, \sigma_{r}$ is a value estimated from standard deviation of blank samples, $b_{k}$ value is a vector of the regression coefficient for the $k$ analytes and $k$ is a number of components in a mixture.

The FSQ technique during a determination of $\beta$-lactam antibiotics applies Fourier preprocessing of the entire absorption spectra of the individual $\beta$-lactam analogs with their degradation products at variable concentration to calculate matrix calibration coefficients.

\subsection{Derivative spectrophotometry}

A derivative spectrophotometry using derivatives of absorbance with respect to wavelength (first $\frac{d A}{d \lambda}=f(\lambda)^{\prime}$, second $\frac{d^{2} A}{d^{2} \lambda}=f(\lambda)^{\prime \prime}$; third $\frac{d^{3} A}{d^{3} \lambda}=f(\lambda)^{\prime \prime \prime}$; respectively) is a suitable tool for overcoming the overlapping spectra problem in analysis of many $\beta$-lactam analogs. Possibility of application of derivative spectrophotometry with zero-crossing point is widely used in analysis of all $\beta$-lactam analogs. The direct correlation between order of used derivative spectrophotometry and similarities of chemical structures of nuclei of $\beta$-lactam analogs has not been observed, e.g., both second-derivative and first-derivative were developed for cephem analogs including the same nuclei (Fig. 4). 


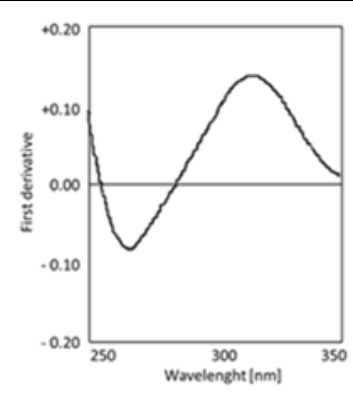

First-derivative spectrum of cefprozil $(1 \mathrm{mg} \%)$ in $0.1 \mathrm{M} \mathrm{HCl}$.

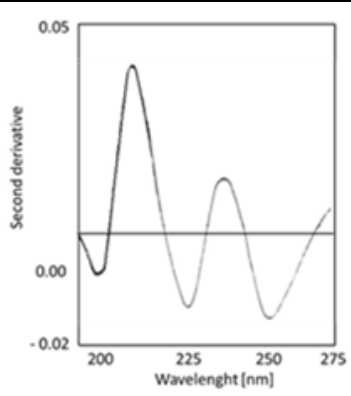

Second-derivative spectrum of cefepime $(20$ $\mu \mathrm{g} / \mathrm{ml})$ in water.

Fig. 4. The application of derivative spectrophotometry for analysis of cephem analogs [15-16].

The application of derivative spectrophotometry for determination of $\beta$-lactam antibiotics was used in the following areas:

- a separation and determination of penam/cephem analogs and inhibitors of $\beta$ lactamase in aqueous solution (e.g., determination of ampicillin sodium in the presence of sulbactam sodium; determination of cefsulodin in the presence of clavulanic acid) [14-15]

- a separation and determination of cephem/carbapenem analog and excipients used in parenteral pharmaceutical dosage forms (e.g., determination of cefepime in the presence of $L$-argininie) [16]

- a separation and determination of cephem analog and its degradation products (e.g., determination of cefprozil in the presence of its degradation products) [17]

- a separation and determination of cephem analog and related compounds from the synthesis (e.g., determination of triethylammonium salt of cefotaxime in the presence of 2-mercaptobenzothiazole) [18]

- a separation and determination of penam/cephem/carbapenem analogs in biological matrix (e.g., determination of amoxicillin, cefuroxime, imipenem in urine) [19].

The separation of often structurally very similar species (e.g., two analogs of cephem, cephem analog and its impurities from synthesis or carbapenem analog and its degradation products) was possible by using derivative spectrophotometry (Fig. 5).

It was proved that the derivative spectrophotometry can be recommended as a method for routine control analysis of pharmaceutical preparation of $\beta$-lactam antibiotics. Derivative spectrophotometry ensured the rapid analysis of parenteral dosage forms and also removed a "background" excipients in oral pharmaceutical dosage forms.

The special potency of derivative spectrophotometry was possibility of its usage in determination of $\beta$-lactam analogs in biological matrix. In this case, to meet the requirements of analytical methods, the selectivity had to be extended in regard with interference of biological endogenous components. It was noticed that the selective determination of penam/cephem/carbapenem in the presence of metabolites (open-ring degradation product) and endogenous substance of urine was possible to achieve. 


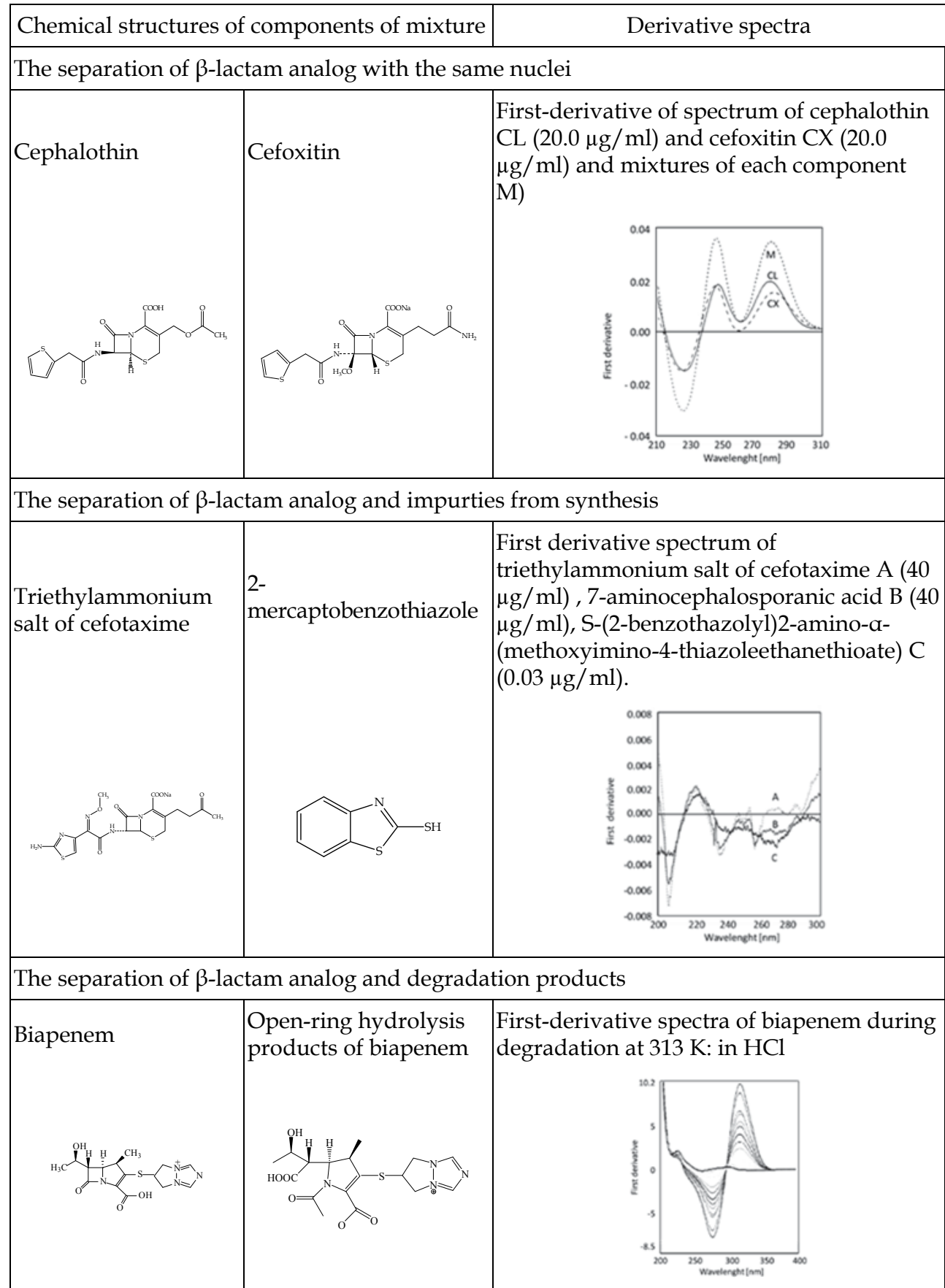

Fig. 5. Separation of some $\beta$-lactam analogs using derivative spectra [18, 20-21]. 


\subsection{Derivative spectrophotometry enriched by chemometric procedures}

The application of chemometric procedures coupled with derivative spectroscopy permits achievement of higher selectivity in determination of $\beta$-lactam antibiotics. Currently, chemometric procedures based on the estimated ratio of spectra derivative for the selective determination of $\beta$-lactam analogs are the most common. It was proved that the application of the ratio of different-order spectra derivatives permitted the separation of binary and tertiary mixtures of $\beta$-lactam antibiotics [22]. During the determination of concentrations of three components (e.g., penicillin-G sodium, penicillin-G procain and dihydrostreptomycin sulphate salts) in a mixture the equation describing the ratio spectra derivative spetrophotometry is as follows:

$$
\frac{d\left(A_{a+b, \lambda} / A_{a, \lambda^{0}}\right)}{d \lambda}=C_{b} \frac{d\left(k_{b, \lambda} / A_{a, \lambda} 0\right)}{d \lambda}+C_{c} \frac{d\left(k_{c, \lambda} / A_{a, \lambda} 0\right)}{d \lambda}
$$

where $A_{a+b+c, \lambda}$ is the absorbance of the ternary mixture of $a, b$ and $c$ at wavelength $\lambda, A_{a, \lambda^{0}}$ is the absorbance of pure component at wavelength $\lambda, C_{b}$ and $C_{c}$ - are the concentrations of $b$ and $c, k_{b, \lambda}$ and $k_{c, \lambda}$ are the products of the molar absorption coefficient of $b$ at wavelength $\lambda$ and the thickness of the absorption cell. Equation 2 is divided by $\mathrm{C}_{b}$ while divisor can be any component of ternary mixture (Fig. 6):

$$
\frac{d\left(A_{a+b, \lambda} / A_{a, \lambda^{0}}\right)}{d\left(A_{b, \lambda} 0 / A_{a, \lambda}\right)}=\frac{C_{b}}{C_{b}^{0}}+\left(C_{c} d \frac{d\left(k_{c, \lambda} / A_{a, \lambda} 0\right)}{d\left(A_{b, \lambda} 0 / A_{a, \lambda} 0\right)}\right)
$$

Equation 3 is drawn:

$$
J=C_{c} d\left(d\left(\frac{d\left(k_{c, \lambda} / A_{a, \lambda} 0\right)}{d\left(A_{b, \lambda} / A_{a, \lambda} 0\right)}\right)\right) / d \lambda
$$

Finally, after the next derivation $\mathrm{J}$ (as the left side of equation 3 ), is proportional to the $\mathrm{C}_{\mathrm{c}}$ value and can be used to determine concentration of component in the ternary mixture (when $A_{a, \lambda^{0}}$ and $A_{b, \lambda^{0}}$ are fixed) [23].

Depending on chemometric procedure, the selective determination of following analogs was possible:

- a separation and determination of carbapenem and degradant (e.g., the determination of ertapenem and its degradant) when the substraction technique was used [24-25]

- a separation and determination of penam and cephem analogs (e.g., the determination of penicillin-G, penicillin-G procain in the presence of dihydrostreptomycin sulphate salts or the determination of cefotaxime and cefadroxil), cephem analog and inhibitor of $\beta$-lactamases (e.g., the determination of cephradine and clavulanic acid) and carbapenem analog and degradation products (e.g., the determination of meropenem and its degradant) when ratio spectra of derivative with all orders were used [26-29]

- a separation and determination of carbapenem and degradation products (e.g., the determination of ertapenem) when the substraction technique was used [30]

- a separation and determination of carbapenem and degradation products (e.g., the determination of ertapenem) when the Kraise's method was used [31]

- a separation and determination of penam analogs (e.g., the determination of ampicillin and flucloxacillin) when multivariate methods (classical least squares and principle component regression) were used. 


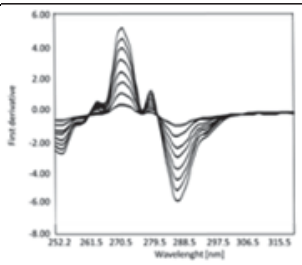

First order derivative ratio spectra of dicloxacillin sodium 50.0-400.0 $\mathrm{mg} / \mathrm{l} \mathrm{using} \mathrm{of} 68.47 \mu \mathrm{g} / \mathrm{ml}$ ampicillin sodium as a divisor [26]

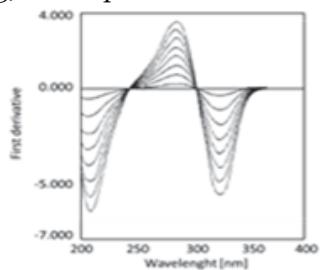

First order derivative ratio spectra of meropenem 4.0-60.0 $\mu \mathrm{g} / 1 \mathrm{using}$ of $32 \mu \mathrm{g} / \mathrm{ml}$ degradate as a divisor [29]

Fig. 6. The application of ratio spectra of derivative spectrophotometry in analysis of $\beta$ lactam antibiotics.

\section{Visible spectrophotometric methods for determination of $\beta$-lactam antibiotics}

The $\beta$-lactam analogs itselfs do not absorb in visible region of radiation. However, many visible spectrophotometric methods were developed for the determination of $\beta$-lactam antibiotics using the effect of formation of "species" giving signals in visible region as the result of chemical derivatization (Fig. 7).

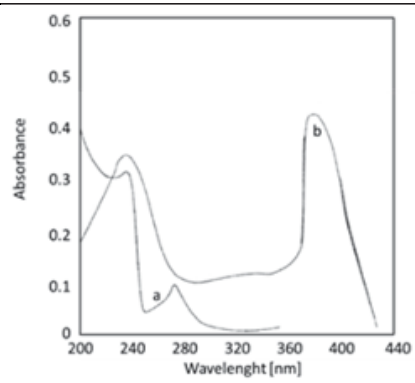

Absorption spectra of amoxicillin trihydrate

(a) $30 \mu \mathrm{g} / \mathrm{ml}$; (b) after reaction with Fe (III) [31]

Fig. 7. The application of derivatization for detetmination of penam analog.

Formation of "species" absorbing visible radiation can be a result of reactions of chemical reagents with:

- $\quad \beta$-lactam analog 
- degradation product of some $\beta$-lactam analog.

Above-mentioned methods were described only for the determination of penam and cephem analogs. The species, absorbing visible radiation, used in analysis of $\beta$-lactam analogs were formed as a result of the following reactions:

- $\quad$ redox

- complexation of metals

- complexation on the base of charge-tranfer process

- formation of ion pairs

- coupling with specified reagents.

\subsection{Visible spectrophotometric methods based on redox reactions}

The methods based on the selective oxidation were reported for penam and cephem analogs containing phenolic substitutes at C6 and C7, respectively. These methods permitted also selective determination of $\beta$-lactam analogs in the presence of excipients being in their pharmaceutical preparations. The application of oxidation properties of iron ions was used in analysis of a huge number of $\beta$-lactam analogs:

- when, as the result of direct reaction with Fe(III) in acidic medium, yellow coloured products $\left(\lambda_{\max }=397 \mathrm{~nm}\right)$ were produced (cefoperazone sodium, cefadroxil monohydrate, cefprozil anhydrous, amoxicillin trihydrate). A possible mechanism of reaction is presented in Fig. 8 [32].

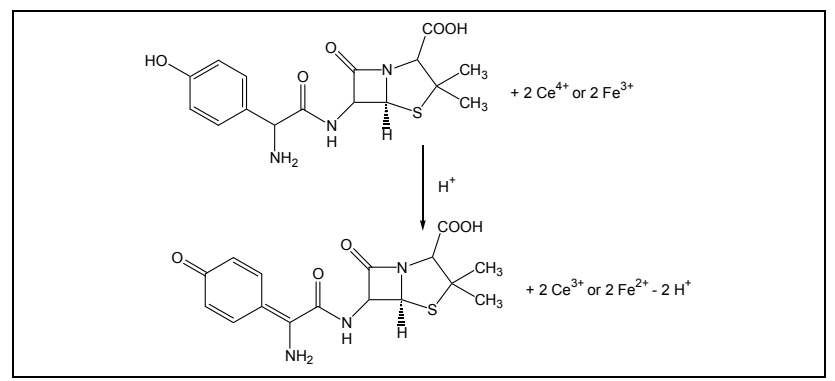

Fig. 8. The mechanism of reaction of amoxicillin and Fe(III)[32].

- when, as the result of indirect reaction, red complex Fe-(o-phen) $)_{2 / 3}\left(\lambda_{\max }=510 \mathrm{~nm}\right)$ was produced. This complex is formed between o-phenanthroline and $\mathrm{Fe}(\mathrm{II})$ which previously was reduced from Fe(III) as the result of oxidation of $\beta$-lactam analogs in alkali medium [33].

The reduction of oxidized quercetin by cephem analogs was used in development of visible spectrophotmetric method for determination of $\beta$-lactam analogs. Quercetin is a flavonol $\left(3,5,7,3^{\prime}, 4^{\prime}\right.$ - pentahydroxyflavone) which is oxidized by $N$-bromosuccinimide giving reddish green colour $\left(\lambda_{\max }=510 \mathrm{~nm}\right)$. As the result of reduction of oxidized form of quercetin by cephem analog fade colour was observed (Fig. 9). This colour is the result of formation of $o-$ quinone derivative of quercetin under the mild oxidants [34]. 


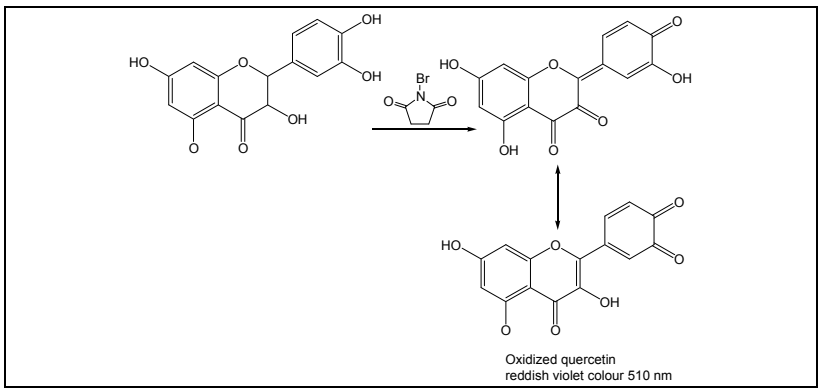

Fig. 9. The mechanism of reduction of quercetin oxidation with $N$-bromosuccinimide [34].

Also, another reagent which is able to oxidize some sulphur atoms present in compounds such as cefotaxime and cefuroxime, is 1-chlorobenzotiazol. As the result of reaction of cephem analogs and 1-chlorobenzotiazol, a product with yellow colour is formed, absorbing radiation at $\lambda_{\max }=298 \mathrm{~nm}$. The suggested possible reaction pathways and absorption spectra are shown in Figure 10 [35].

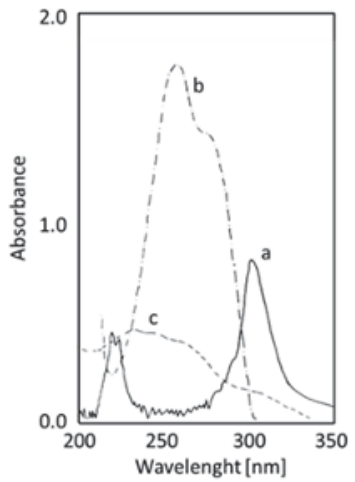

Absorption spectra of $65 \mu \mathrm{g} / \mathrm{ml}$ cefotaxime sodium (c),

1-chlorobenzotiazol $(0.002 \mathrm{M})(\mathrm{b})$ and their reaction product (a).

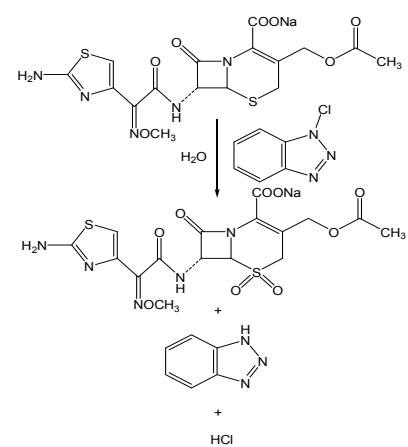

The schemes of reaction between cefotaxime sodium and 1-chlorobenzotiazol

Fig. 10. The mechanism of reaction of cephem analog and 1-chlorobenzotiazol [35].

In indirect spectrophotometry, redox properties of iodine were used in determination of penam analog (amplicillin, penicillin V, amoxicillin, cloxacillin) and cephem analogs (cefadroxil, ceftezoxime). The method based on formation of hypoiodite, from excess of iodine (which did not react with $\beta$-lactam analog) under alkaline conditions. Hypoiodite reduced the intensity of wool fast blue colour (5,9-dianilo-7-phenyl 4,10disulphbenzo[a]phenazinium hydroxide) by disruption of phenazine chromophore [36].

Especially, for cefadoxil many visible spectrophotometric methods based on redox reactions were proposed (Fig. 11). These methods were based on the reaction with different oxidizing reagents: 
- 4-aminoantipyrine in the presence of potassium hexacyanoferrate(III) in alkaline medium $\left(\lambda_{\max }=505 \mathrm{~nm}\right.$ [37]

- 3-methyl-2-benzothiazolinone hydrozone hydrochloride in the presence of ceric ammonium sulphate $\left(\lambda_{\max }=410 \mathrm{~nm}\right)$ [38]

- 4-aminophenazone in the presence of potassium hexacyanoferrate (III) $\left(\lambda_{\max }=510 \mathrm{~nm}\right)$

- 2,6-dichloroquinone-4-chlorimide (Gibb's reagent) $\left(\lambda_{\max }=620 \mathrm{~nm}\right)$ [39]

- $\quad N$-bromosuccinimide or $N$-chlorosuccinimide in alkali medium $\left(\lambda_{\max }=395 \mathrm{~nm}\right)$ [40]

- $\quad$ sodium persulfate in alkaline medium $\left(\lambda_{\max }=350 \mathrm{~nm}\right)$ [41]

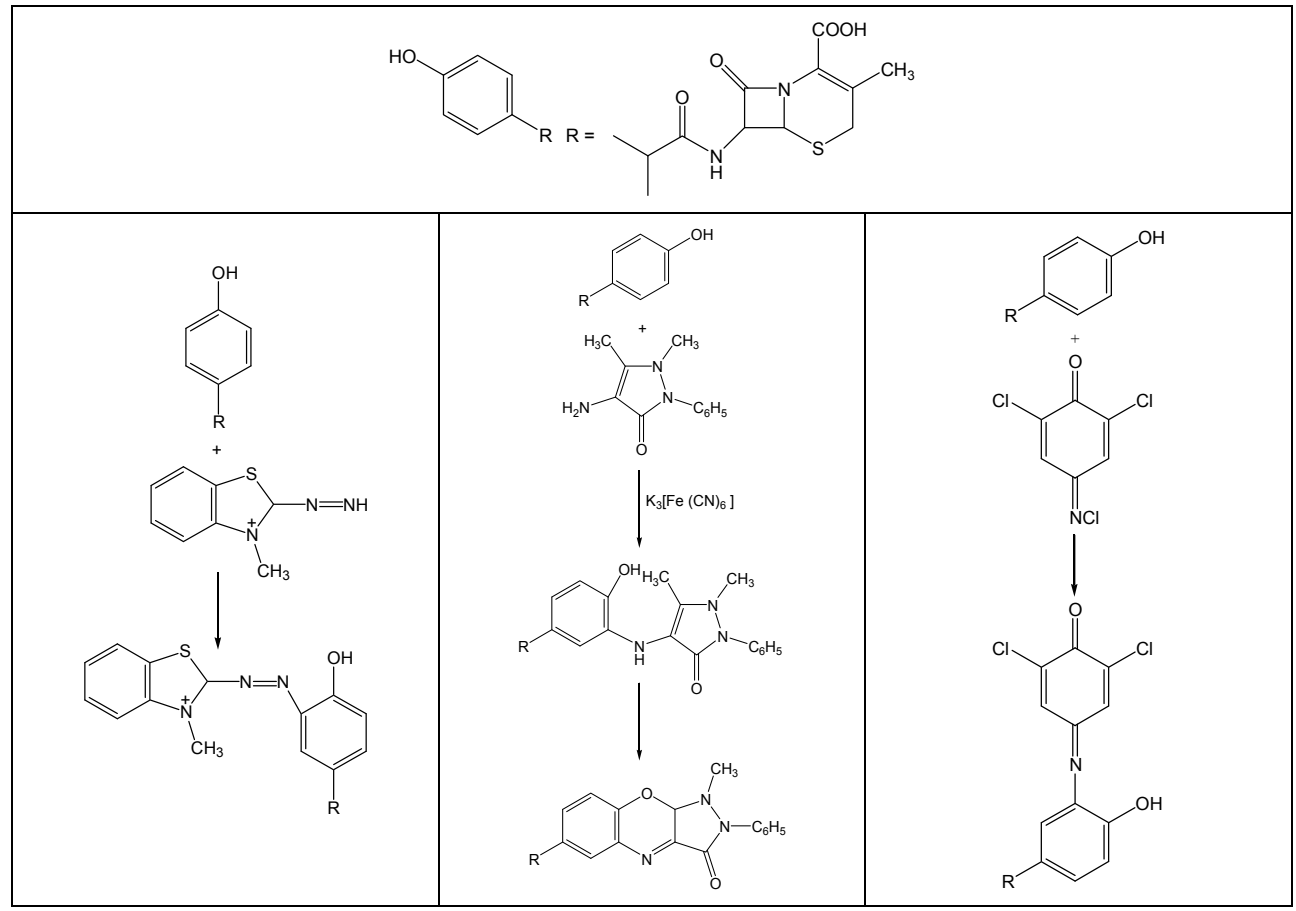

Fig. 11. Schemes of some oxidation reactions of cefadoxil [39].

\subsection{Visible spectrophotometric methods based on formation of complex with metal}

The formation of colored complexes as a consequence of the interactions between metal ions and analytes (penam and cephem analogs) resulted from:

- $\quad$ a direct reaction between $\beta$-lactam analogs and metal ions

- $\quad$ a reaction between the degradation products of $\beta$-lactam analogs and metal ions.

Direct complexation of $\beta$-lactam analogs and metal ions with formation of yellowish-brown chelate complex was possible due to the presence of suphur atoms in the $\beta$-lactam ring and the thiazole ring. Cephem analogs (cefpodoxime, ceftizoxime, ceftazidime, ceftiaxone and cefixime) gave with palladium(II) ions, absorbing complex, in the presence of sodium lauryl sulphate as surfactant, in the range 300-500 nm [42]. B-lactam analogs containing the phenolic ring with free ortho position to the hydroxyl group (amoxicillin trihydrate, 
cefperazone sodium, cefadroxil monohydrate, cefprozil anhydrous) reacted with nitrous acid forming the nitroso derivatives. They were capable of tautometric interconversions to form colored complex in the presence of copper(II) ions (Fig. 12). The stoichiometric ratios (nitroso derivative to copper(II)) were determined by the Job's method 2:1 [43].

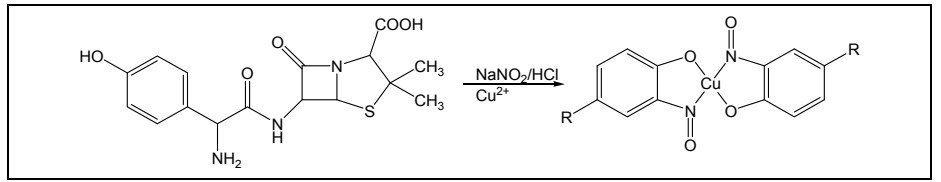

Fig. 12. Scheme of the reaction of nitrous acid, $\mathrm{Cu}(\mathrm{II})$ with phenolic $\beta$-lactam analogs [43].

The determination of cephalexin, cefixime, ceftriaxone, cefotaxime based on the Bent-French method, in which degradation products of $\beta$-lactam analogs with metal ions form the colored complexes, was developed. Hydroxamic acids formed by hydroxiaminolysis of cephem analogs (1:3), formed complexes with iron (II) ions (Fig. 13) [44].

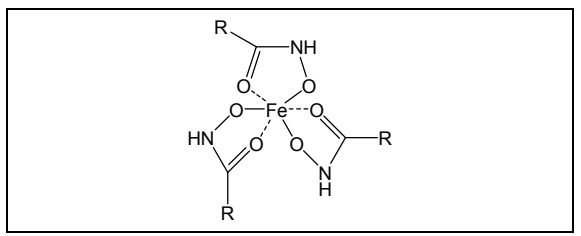

Fig. 13. The scheme of hydroxamic acid-iron(III) [44].

\subsection{Visible spectrophotometric methods based on formation of charge-transfer complex}

Some drugs, including penam and cephem analogs, are electron donors. Therefore, they form charge-transfer complexes with compounds that are $\sigma-$ and $\Pi$-accepptors of electrons. The wavelengths at which the absorption maxima of charge-transfer complexes of $\beta$-lactam antibiotics were measured depended on what of reagent was used the acceptor:

- $\quad p$-chloranilic acid gives coloured complex species at 520-529 nm during analysis of cefotaxime sodium, cefuroxime sodium, ceftazidime pentahydrate, cephalexin monohydrate, cefotaxime sodium, cephradine, cephaloridine sodium, cefoperazone sodium (ratio 1:1); cephalotin sodium, cefixime, cefprozil anhydrous, cefazolin sodium, cephapirin (ratio 1:2); cefaclor (ratio 1:4) [45]

- $\quad p$-nitrophenol, 2,4-dinitrophenol, 3,5-dinitrosalycilic acid, picramic acid and picric acid give greenish yellow complexes at 446, 435, 442, 473 and $439 \mathrm{~nm}$, respectively during determination of flucloxacillin (ratio 1:1) [46]

- 7,7,8,8-tetracyanoquinodimethane (TCNQ) gives coloured complex species at 838-843 $\mathrm{nm}$ during analysis of cefotaxime sodium, cefuroxime sodium, cephapirin sodium, cefazoline sodium, cephalexin monohydrate, cefadroxil monohydrate, cefoperazone and ceftazidime (ratio 1:1)[47]

- 2,3-dichloro-5,6-dicyano- $p$-benzo-quinone (DDQ) gives coloured complex species at 460 $\mathrm{nm}$ during analysis of cephapirin sodium, cefazoline sodium, cephalexin monohydrate, cefadroxil monohydrate, cefoperazone and ceftazidime (ratio 1:1) [47] 
- $\quad$ iodine gives coloured complex species at 838-843 nm during analysis of cephapirin sodium, cefazoline sodium, cephalexin monohydrate, cefadroxil monohydrate, cefoperazone and ceftazidime (ratio 1:1) [47].

During formation of charge-transfer complexes between $p$-chloranilic acid and d-donors electrons (A) from group of cephem analogs (D) in polar solvents the radical anion is formed.

$$
\mathrm{D}+\mathrm{A} \underset{\text { complex }}{\rightleftharpoons}(\mathrm{D}---\mathrm{A}) \stackrel{\text { polar sovent }}{\rightleftharpoons} \dot{\mathrm{D}}^{+} \mathrm{A}^{\cdot-}
$$

Electron transfer from donor to the acceptor moiety occurred with the formation of intensely coloured radical ions with high molar absorptivity value. The formation of charge-transfer complex of some cephem analogs (D) with iodine in 1,2-dichloroethane (J) is observed with the change of colour from violet to lemon yellow:

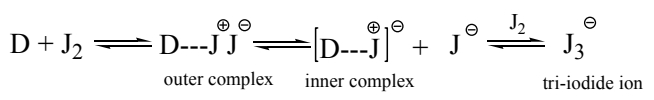

Only in 1,2-dichloroethane, formation of tri-iodide ion pair (inner complex), showing two absorption maxima at $290 \mathrm{~nm}$ and $364 \mathrm{~nm}$, was possible. This complex originated from an early intermediate outer complex D--- $\mathrm{J}_{2}$. While the interactions of some cephem analogs with DDQ and TCNQ took place according to the following simple relationship:

$$
\mathrm{D}+\mathrm{A} \underset{\text { complex }}{\rightleftharpoons}(\mathrm{D}---\mathrm{A})
$$

\subsection{Visible spectrophotometric methods based on formation of ion pair}

In visible spetrophotometric analysis of penam and cephem analogs, their ability to form ion-pair was also used. The penam analogs contacting the tertiary amine group (ampicillin, dicloxacillin, flucloxacillin, amoxicillin) and $\mathrm{Mo}(\mathrm{V})$-thiocyanate binary complex in hydrochloric acid give coloured ion-pair formation absorbing at $\lambda=467 \mathrm{~nm}$ (Fig. 14) [48].

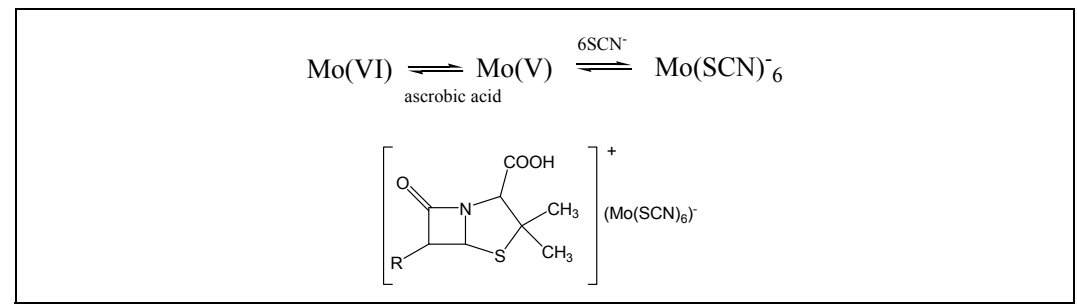

Fig. 14. Scheme of Mo(V)-thiocyanate- $\beta$-lactam ion-pair [48].

Some cephem analogs (cephaprin sodium, cefuroxime sodium, cefotaxime sodium, cefoperazone sodium, cefadroxil, ceftazidime, cefazolin sodium and cefaclor) can be determined spectrophotometrically based on formation of ion-pair complex with ammonium reinecekate. In acidic medium at $25 \pm 2{ }^{\circ} \mathrm{C}$, as the reaction products, the complex, absorbing at $525 \mathrm{~nm}$, was formed according to the scheme [49]:

cephem analog hydrochloride + ammonium reineckate $\rightarrow$ cephem reineckate + ammonium chloride 


\subsection{Visible spectrophotometric methods based on coupling with specified reagents}

The analysis of phenolic derivatives of penam and cephem analogs were possible by measurement of absorption species formed as a result of reations with specified reagents.

Diazo coupling of $\beta$-lactam analogs was conducted with the following compounds:

- benzocaine in thrietylamine medium for determination of cefadroxil and amoxicillin. Stoichiometric ratio of formed species was $1: 1$ with peaking at $\lambda=455 \mathrm{~nm}$ and $\lambda=442 \mathrm{~nm}$, respectively [50] .

- electron-deficient polinitro derivatives for determination of amoxicillin, cefoperazone, cefadroxil, cefprozil. Complexes of the Meisenheimer type were formed [51].

Suggested mechanisms of coupling reaction of phenol derivative of $\beta$-lactam analogs were presented in Fig. 15.

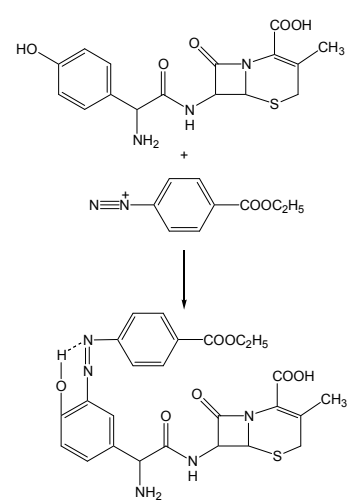

Reaction of diazo coupling of cefadroxil
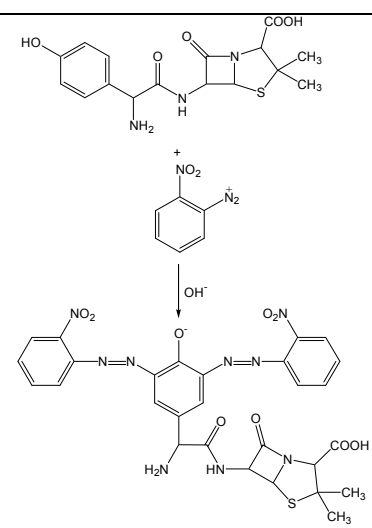

Reaction of diazo coupling of amoxicillin

Fig. 15. Schemes of reactions of coupling of $\beta$-lactam analogs [50-51].

For determination of phenolic derivative of $\beta$-lactam analog (cefadroxil) measurement of absorption of formed product in the reaction between it and 4-aminoantipyrine in the presence of alkaline potassium hexacyanoferrate(III) at $510 \mathrm{~nm}$ was also proposed (Fig. 16). Potassium hexacyanoferrate(III), being oxidant in this reaction, yielding $N$-substituted quinine imines and in the result was responsible for formation of red-colored antipyrine dye. Additionally, a sequential injection analysis (SIA) spectrophotometric procedure for the determination was reported [52].

1,2-naphthoquinone-4-sulfonic acid is the reagent permitting the nucleophilic substitution reaction in area of amino group of penem (amoxicillin) and cephem (cephalexin) analogs (Fig. 17). The stoichiometric ratio of these species was $1: 1$ and they absorb at $\lambda=463 \mathrm{~nm}$ [53].

The extension of the methodology for determination of cephalexin by using the H-point standard additions method (HPSAM) and the generalized H-point standard additions methods (GHPSAM) (after solid phase extraction cartridges) permitted also its analysis in urine [53]. 


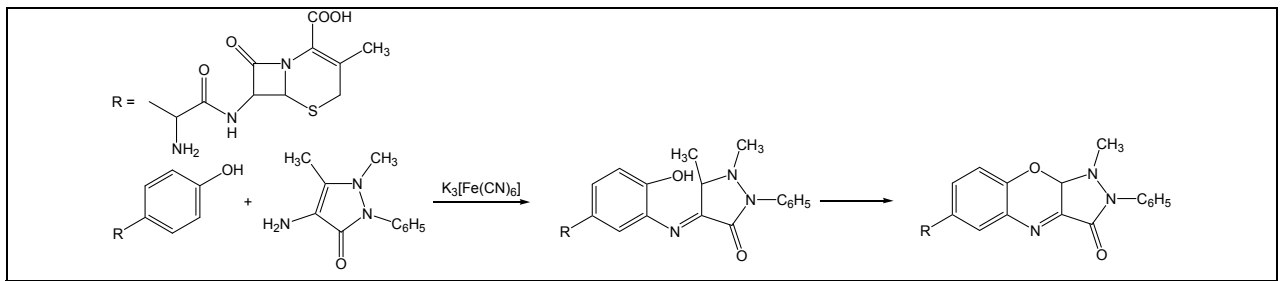

Fig. 16. The reaction mechanism of cefadroxil with 4-aminoantipyrine in the presence of alkaline $\left[\mathrm{Fe}(\mathrm{CN})_{6}\right]^{3-}$

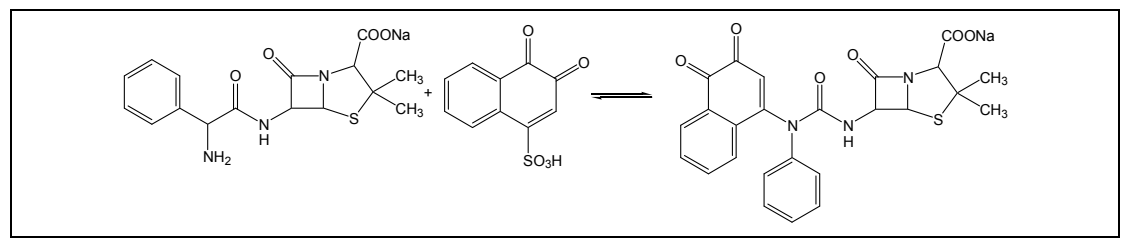

Fig. 17. The reaction mechanism of ampicillin sodium and 1,2-naphthoquinone-4-sulfonic acid [53].

\subsection{Visible spectrophotometric methods based on degradation products of $\beta$-lactam analogs}

The significant instability of $\beta$-lactam analogs was also exploited in the spectrophotometrical determination of $\beta$-lactam analogs in the visible region.

As the intermediate stages of determination, depending on the affecting factors, the following were present:

- degradation products typical of an acidic environment

- degradation products characteristic of a basic environment.

The formation of degradation products of $\beta$-lactam analogs in conditions of a basic hydrolysis was also the first stage during development of visible spectrophotometric methods. As the result of reactivity of degradation products formed in basic medium with some reagents, the determination of the following $\beta$-lactam analogs was possible:

- cephem analogs (cefadroxil, cefotaxime), when coupling factor were $N, N$-diethyl-pphenylenediamine sulfate and $\mathrm{Fe}(\mathrm{III})(\lambda=670 \mathrm{~nm})$ or $p$-phenylenediamine dihydrochloride and Fe(III) $(\lambda=597 \mathrm{~nm})$ [54]

- cephem analogs (cefotaxime, ceftriaxone, cefradine) when reducing factor was potassium iodate (required acidic medium) and a result of the reaction was colour change of leuco crystal violet under the influence of formed iodine $(\lambda=588 \mathrm{~nm})$ [55]

- cephem analogs (cefotaxime sodium) when coupling factor was 1,10-phenanthroline and ferric chloride $(\lambda=520 \mathrm{~nm})$ [56]

- $\quad$ penam analogs (amoxicillin, ampicillin) and cephem analogs (cephalexin, cephradine) when reducing factor of formed hydrolyzed products was $\mathrm{J}_{2}$ (required acidic medium) $(\lambda=460 \mathrm{~nm})[57]$. 
The significant expansion of possibilities for the developed analytical method was the usage of flow injection analysis (FIA).

Similarly, in the case of determination of penam analysis during acidic hydrolysis $(1.0 \mathrm{M}$ $\mathrm{HCl}$ ), formation of non-absorbing degradation products was the intermediate stage of their analysis. Complex which is necessary for achievement of spectrophotometric signals, was formed between penicillamine and palladium(II) chloride, peak at $334 \mathrm{~nm}$ (Fig. 18) [58].

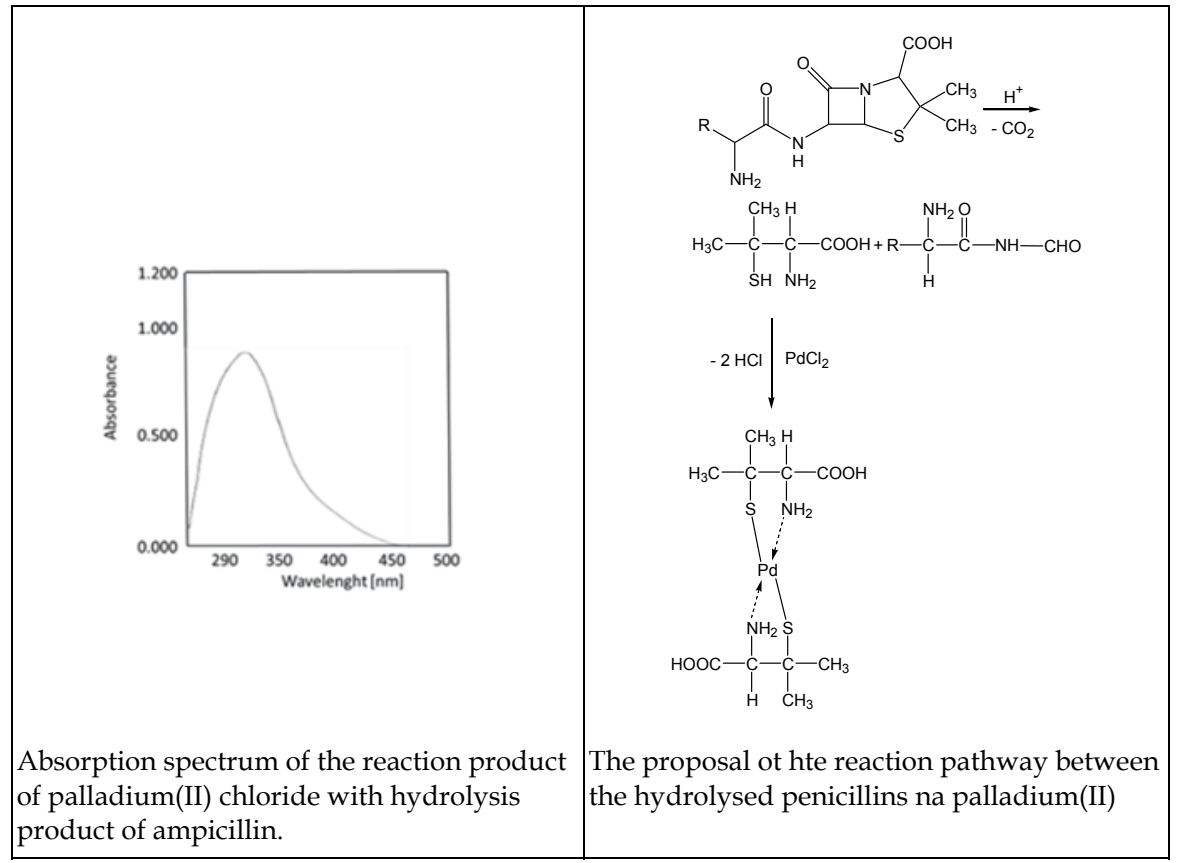

Fig. 18. Absorption spectra based on reaction of degradation products of penam analogs and palladium (II) and suggested mechanism of the reaction [58].

In conditions of acidic hydrolysis, determination of cephem analogs took place. Vanadium(IV) after reduction from vanadium(V), reacted with forming degradation products. Colored complexes of some cephem analogs (cephalexin, cephaprine sodium, cefazolin sodium, cefotaxime) were found, absorbing at 515, 512, 518 and $523 \mathrm{~nm}$, respectively (Fig. 19) [59] 


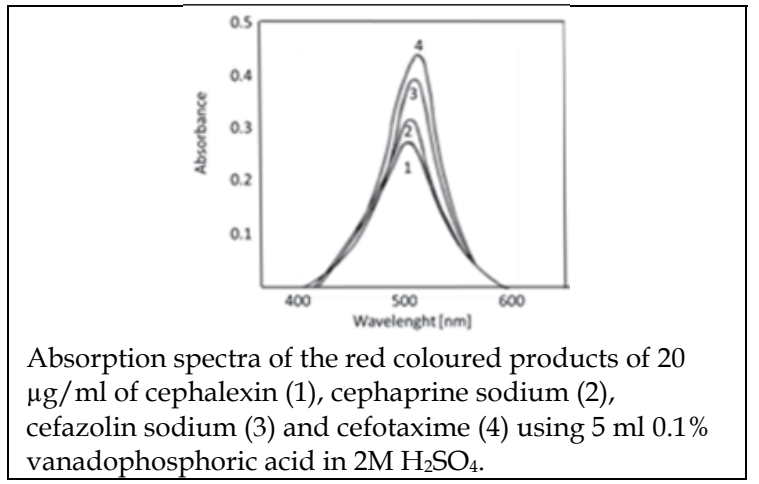

Fig. 19. Absorption spectra based on reaction of degradation products of cephem analogs and vanadium(IV) [59].

\section{Conclusion}

Spectrophotometric determinations of drugs in Vis-UV region are ones from simpler and cheaper methods used in quantitative pharmaceutical analysis. The key problem in application of these methods in analysis of multicomponents mixture is achievement of desired selectivity. Above-mentioned procedures shown that using various methods of chemical derivatization (significant for analysis in Vis region) or ones based on mathematical extends (significant for analysis in UV region) the selective determination of such labile drug as $\beta$-lactam antibiotics was possible. Taking into consideration variability of stability of some $\beta$-lactam analogs (in the presence of impurities, degradation products, inhibitors of $\beta$-lactamases, excipients and other drugs) and pathways of their degradation, confirmation of selectivity of method and values of other validation parameters for each analogs is required. It is important that analytical method used as alternative, referential one should not use spectrophotometric properties of compound as the base of detection. Recently, the development of chemometric procedures, as the complementary method for spectrophotometric ones, is often supported by suitable, high level software and equipment of apparatus allowing to assume that analysis based on calculated algorithms meet all validation criteria and can replace these methods which require pre-treatment of samples. Moreover, these methods permit multiple determination of the same sample and can be used in routine control analysis of intravenous pharmaceutical dosage forms. Additionally, proposed conditions of determination using spectrophotometric methods are in accordance with all criteria identified as the trends of "green chemistry". A enrichment of visible spectrophotometric methods by the application of FIA and SIA permit accept of challenges of modern industrial-scale pharmaceutical analysis [60]. It can be expected that spectrophotometric methods enriched by chemometric procedures will become an important field of pharmaceutical analysis including also labile drugs.

\section{Acknowledgment}

The authors thank the State Committee for Scientific Research, Poland, for project N N405 683040 . 


\section{References}

[1] Cielecka-Piontek J. Michalska K. Zalewski P. Jelińska A. Recent Advances in stability studies of carbapenems. Current Pharmaceutical Analysis 2011;7 213-227.

[2] El-Shaboury S. Saleh G. Mohamed F. Rageh A. Analysis of cephalosporin antibiotics. Journal of Pharmaceutical and Biomedical Analysis.2007;45 1-19.

[3] Cielecka-Piontek J. Michalska K. Zalewski P. Zasada S. Comparative review of analytical techniques for determination of carbapenems. Current Analytical Chemistry 2012; 8 91-115.

[4] ICH. Stability testing of new drug substances and products. In: Proceedings of International conference on Harmonization. Geneva: IFPMA; 2000.

[5] Parisotto G. Ferrao M. Furtado J. Molz R. Determination of amoxicillin content in powdered pharmaceutical formulations using DRIFTS and PLS. Brazilian Journal of Pharmaceutical Sciences 2007;43(1) 89-95.

[6] Tabelbpour Z. Tavallaie R. Agmadi S. Abdollahpour A. Simultanesous determination of penicillin $G$ salts by infrared spectroscopy: Evaluation of combining orthogonal signal correction with radial basis function-partial least squares regression. Spectrochimica Acta Part A 2010;76 452-457.

[7] European Pharmacopoeia 7th ed. 2010

[8] Ivama V. Rodrigues L. Guaratini C. Zanoni M. Spectrophotometric determination of cefaclor in pharmaceutical preparations. Quimica Nova 1999;22(2) 1-6.

[9] Deshpande A. Baheti K. Chatterjee N. Degradation of $\beta$-lactam antibiotics. Current Science 2004;78(12) 1684-1695.

[10] Rojanarata T. Opanasopit P. Ngawhirunpat T. Saehuan Ch. Wiyakrutta S. Meevootisom V. A simple sensitive and green bienzymatic UV-spectrophotometric assay for amoxicillin formulations. Enzyme and Microbial Technology 2010;46 292-296.

[11] Campins-Falco P. Sevillano-Cabeza A. Gallo-Martinez L. Bosch-Reig F. MonzoMansanet I. Comparative Study on the Determination of Cephalexin in its Dosage Forms by Spectrophotometry and HPLC with UV-vis Detection. Microchimica Acta 1997;126 207-215.

[12] Cantarelli M. Pellerano R. Marchevsky E. Camina J. Simultaneous Determination of Amoxicillin and Diclofenac in Pharmaceutical Formulations Using UV Spectral Data and the PLS Chemometric Method. Analytical Sciences 2011;27 73-78.

[13] Abdel-Hamid M. FSQ spectrophotometric and HPLC analysis of some cephalosporins in the presence of their alkali-induced degradation products. Il Farmaco 1998;53 132-138.

[14] Mahgoub H. Aly F. Uv-spetrophotometric determination of ampicillin sodium and sulbactam sodium in two-component mixtures. Journal of Pharmaceutical and Biomedical Analysis 1998;17 1273-1278.

[15] Murillo J. Lemus J. Garcia L. Simultaneous determination of the binary mixtures of cefsulodin and clavulanic acid by using first-derivative spectrophotometry. Journal of Pharmaceutical and Biomedical Analysis 1995;13(6) 769-776.

[16] Rodenas V. Parra A. Garcia-Villanova J. Gomez M. Simultaneous determination of cefepime and L-arginine in injections by second-derivative spectrophotometry. Journal of Pharmaceutical and Biomedical Analysis 1995;13 1095-1099.

[17] Daabees H. Mahrous M. Abdel-Khalek M. Beltagy Y. Emil K. Spectrophotometric determination of cefprozil in pharmaceutical dosage forms in urine and in the 
presence of its alkaline induced degradation products. Analytical Letters 2001;34(10) 1639-1655.

[18] Nuevas L. Gonzalez R. Rodriguez J. Hoogmartens J. Derivative spectrophotometric determination of the thriethylammonium salt of cefotaxime in presence of related compound from the synthesis. Journal of Pharmaceutical and Biomedical Analysis 1998;18 579-583.

[19] Forsyth R. Ip D. Determination of imipenem and cilastatin sodium in Primaxin $®$ by first order derivative ultraviolet spectrophotometry. Journal of Pharmaceutical and Biomedical Analysis 1994;12 1243-1248.

[20] Murillo J. Lemus J. Garcia L. Analysis of binary mixtures of cephalothin and cefoxitin by using first-derivative spectrophotometry. Journal of Pharmaceutical and Biomedical Analysis 1996;14 257-266.

[21] Cielecka-Piontek J. Lunzer A. Jelińska A. Stability-indicating derivative spectrophotometry method for the determination of biapenem in the presence of its degradation products. Central European Journal of Chemistry 2011;9 35-40.

[22] Mohamed A. Salem S. Maher E. Chemometrics-assisted spectrophotometric determination of certain $\beta$-lactam antibiotics combinations. Thaiwan Journal Pharmaceutical Sciences 2007;31 9-27.

[23] Lin Z. Liu J. Chen G. A new method of Fourier-transform smoothing with ratio spectra dervative spectrophotometry. Fresenius Journal Analytical Chemistry 2001;370 997-1002.

[24] Zając M. Cielecka-Piontek J. Jelińska A. Development and validation of UV spectrophotometric and RP-HPLC methods for determination of ertapenem during stability studies. Chemia Analityczna 2006;51 761-768.

[25] Cielecka-Piontek J. Jelińska A. The UV-derivative spectrophotometry for the determination of doripenem in the presence of its degradation products. Spectrochimica Acta Part A 2010;77 554-557.

[26] Morelli B. Determination of ternary mixtures of antibiotics by ratio-spectra zerocrossing first- and third-derivative spectrophotometry. Journal of Pharmaceutical and Biomedical Analysis 1995;13(3) 219-227.

[27] Morelli B. Derivative spectrophotometry in the analysis of mixtures of cefotaxime sodium and cefadroxil monohydrate. Journal of Pharmaceutical and Biomedical Analysis 2003;32 257-267.

[28] Murillo J. Lemus J. Garcia L. Application of the ratio spectra derivative spectrophotometry to the analysis of cephradine and clavulanic acid in binary mixtures. Fresenius Journal Analytical Chemistry 1993;347 114-118.

[29] Elragehy N. Abdel-Moety E. Hassan N. Rezk M. Stability-indicating determination of meropenem in presence of its degradation product. Talanta 2008;77 28-36.

[30] Zając M. Cielecka-Piontek J. Jelińska A. Development and validation of UV spectrophotometric and RP.HPLC methods for determination of ertapenem during stability studies. Chemia Analityczna 2006;51 761-768.

[31] Hassan N. Abdel-Moety E. Elragehy N. Rezk M. Selective determination of ertapenem in the presence of its degradation product. Spectrochimica Acta Part A 2009;72 915921.

[32] Salem H. Saleh G. Selective spectrophotometric determination of phenolic $\beta$-lactam antibiotics. Journal of Pharmaceutical and Biomedical Analysis 2002;28 1205-1213. 
[33] Al-Momani I. Spectrophotometric determination of selected cephalosproins in drug formulations using flow injection analysis. Journal of Pharmaceutical and Biomedical Analysis 2001;25 751-757.

[34] Saleh G. El-Shaboury S. Mahomed F. Rageh A. Kinetic spectrophotometric determination of certain cephalosporins using oxidized quercetin reagent. Spectrochimica Acta Part A 2009;73 946-954.

[35] Ayad M. Shalaby A. Abdellatef H. Elsaid H. Spectrophotometric determination of certain cephalosporins though oxidation with cerium(IV) and 1chlorobenzotriazole. Journal of Pharmaceutical and Biomedical Analysis 1999;20 557-564.

[36] Sastry Ch. Rao S. Naidu P. Strinivas K. New spectrophotometric method for the determination of some drugs with iodine and wool fast blue BL. Talanta 1998;45 1227-1234.

[37] Feng S. Jiang J. Fan J. Chen X. Sequential injection analysis with spectrophotometric detection of cefadroxil and amoxicillin in pharmaceuticals. Chemia Analityczna 2007;52(83) 83-92.

[38] Sastry Ch. Rao K. Prasad D. Determination of cefadroxil by three simple spectrophotometric methods using oxidative coupling reactions. Mikrochimica Acta 1997;126 167-172.

[39] Makchit J. Upalee S. Thongpoon Ch. Liawruangrath B. Liawruangrath S. Determination of cefadroxil by sequential injection with spectrophotometric detector. Analytical Sciences 2006;22 591-597.

[40] Salem G. Two selective spectrophotometric methods for the determination of amoxicillin and cefadroxil. Analyst 1996;121 641-645.

[41] Helaleh M. Abu-Named E. Jamhour R. Spectrophotometric determination of selected cephalosporins. Acta Poloniae Pharmaceutica 1998;55(2) 87-91.

[42] Walily A. Gazy A. Belal S. Khamis E. Quantitative determination of some triazole cephalosporins through complexation with palladium (II) chloride. Journal of Pharmaceutical and Biomedical Analysis 2000;22 385-392.

[43] Salem H. Selective spectrophotometric determination of phenolic $\beta$-lactam antibiotics in pure forms and in their pharmaceutical formulations. Analytica Chimica Acta2004;515 333-341

[44] Eric A. Karljikovic-Rajic K. Vladimirov S. Zivanov-Stakic D. Spectrophotometric determinations of certain cephalosporins using ferrihydroxamate method. Spectroscopy Letters 1997;30(2) 309-313.

[45] Saleh G. Askal H. Darwish I. El-Shorbagi A. Spectroscopic analytical study for the charge-transfer complexation of certain cephalosporins with chloroanilic acid. Analytical Sciences 2003;19 281-287.

[46] El-Mammli M. Spectrophotometric determination of flucloxacillin in pharmaceutical preparations using some nitrophenols as a complexing agent. Spectrochimica Acta Part A 2003;59 771-776.

[47] Saleh G. Askal H. Radwan M. Omar M. Use of charge-transfer complexation in the spectrophotometric analysis of certain cephalosporins. Talanta 2001;54 1205-1215.

[48] Mohamed G. Spectrophotometric determination of amipicillin dicluxacillin flucloxacillin and amoxicillin antibiotic drugs: ion-pair formation with 
molybdenum and thiocyanate. Journal of Pharmaceutical and Biomedical Analysis 2001;24 561-567.

[49] Salem H. Askal H. Colourimetric and AAS determination of cephalosporins using Reineck's salt. Journal of Pharmaceutical and Biomedical Analysis 2002;29 347-354.

[50] El-Ashry S. Belal F. El-Kerdawy M. Wasseef D. Spectrophotometric determination of some phenolic antibiotics in dosage forms Mickrochimica Acta 2000;135 191-196.

[51] Freitas S. Silva V. Araujo A. Canceicao M. Montenegro M. Reis B. Paim P. A multicommuted flow analysis method for the photometric determination of amoxicillin in pharmaceutical formulations using a diazo coupling reaction. Journal of Brazilian Chemistry Society 2011;22(2) 279-285.

[52] Xu L. Wang H. Xiao Y. Spectrophotometric determination of amipicillin sodium in pharmaceutical products using sodium 12 naphtoquinone-4-sulfonic as the chromogentic reagent. Spectrochimica Acta Part A 2004;60 3007-3012.

[53] Gallo-Martinez L. Sevillano-Cabeza A. Campins-Falco P. Bosch-Reig F. A new derivatization procedure for the determination of cephalexin with 12naphtoquinone 4-sulphonate in pharmaceutical and urine samples using solidphase extraction cartridges and UV-visible detection. Analytica Chimica Acta 1998;370 115-123.

[54] Metwally F. Alwarthan A. Al-Tamimi S. Flow-injection spectrophotometric determination of certain cephalosporins based on the formation of dyes. Il Farmaco 2001;56 601-607.

[55] Buhl F. Szpilkowska-Sroka B. Spectrophotometric determination of cephalosporins with Leuno crystal violet Chemia Analityczna 2003;48(145) 145-149.

[56] Rao G. Kumar K. Chowdary P. Spectrophotometric methods for the determination of cefotaxime sodium in dosage forms. Indian Journal of Phamraceutical Sciences 2001;63(2) 161-163.

[57] Al-Momani I. Flow-injection spectrophotometric determination of amoxicillin cephalexin ampicillin and cephradine in pharmaceutical formulations. Analytical Letters 2004; 37(10) 2099-2110.

[58] Belal F. El-Kerdawy M. El-Ashry S. El-Wasseef D. Kinetic spectrophotometric determination of ampicillin and amoxicillin in dosage forms. Il Farmaco 2000;55 680-686.

[59] Amin A. Shama S. Vanadophosphoric acid as a modified reagent for the spetrophotometric determination of certain cephalosporins and their dosage forms Monatsefte fur Chemie 2000;131 313-319.

[60] Tzanavaras P. Themelis D. Review of recent applications of flow injections spectrophotometry to pharmaceutical analysis. Analytica Chimica Acta 2007;588 1-9. 


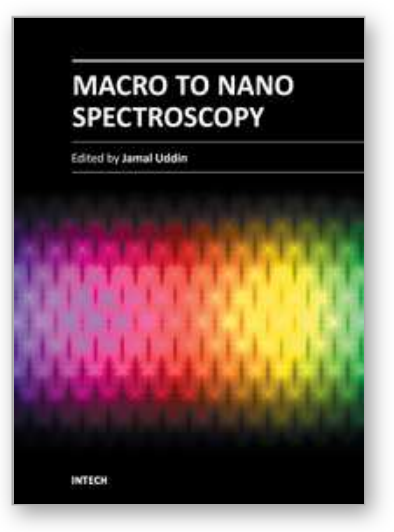

\author{
Macro To Nano Spectroscopy \\ Edited by Dr. Jamal Uddin
}

ISBN 978-953-51-0664-7

Hard cover, 448 pages

Publisher InTech

Published online 29, June, 2012

Published in print edition June, 2012

In the last few decades, Spectroscopy and its application dramatically diverted science in the direction of brand new era. This book reports on recent progress in spectroscopic technologies, theory and applications of advanced spectroscopy. In this book, we (INTECH publisher, editor and authors) have invested a lot of effort to include 20 most advanced spectroscopy chapters. We would like to invite all spectroscopy scientists to read and share the knowledge and contents of this book. The textbook is written by international scientists with expertise in Chemistry, Biochemistry, Physics, Biology and Nanotechnology many of which are active in research. We hope that the textbook will enhance the knowledge of scientists in the complexities of some spectroscopic approaches; it will stimulate both professionals and students to dedicate part of their future research in understanding relevant mechanisms and applications of chemistry, physics and material sciences.

\title{
How to reference
}

In order to correctly reference this scholarly work, feel free to copy and paste the following:

Judyta Cielecka-Piontek, Przemysław Zalewski, Anna Krause and Marek Milewski (2012). Spectrophotometric Methods as Solutions to Pharmaceutical Analysis of $\beta$-Lactam Antibiotics, Macro To Nano Spectroscopy, Dr. Jamal Uddin (Ed.), ISBN: 978-953-51-0664-7, InTech, Available from:

http://www.intechopen.com/books/macro-to-nano-spectroscopy/spectrophotometric-methods-as-solutions-topharmaceutical-analysis-of-lactam-antibiotics

\section{INTECH}

open science | open minds

\section{InTech Europe}

University Campus STeP Ri

Slavka Krautzeka 83/A

51000 Rijeka, Croatia

Phone: +385 (51) 770447

Fax: +385 (51) 686166

www.intechopen.com

\section{InTech China}

Unit 405, Office Block, Hotel Equatorial Shanghai

No.65, Yan An Road (West), Shanghai, 200040, China

中国上海市延安西路65号上海国际贵都大饭店办公楼 405 单元

Phone: +86-21-62489820

Fax: $+86-21-62489821$ 
(C) 2012 The Author(s). Licensee IntechOpen. This is an open access article distributed under the terms of the Creative Commons Attribution 3.0 License, which permits unrestricted use, distribution, and reproduction in any medium, provided the original work is properly cited. 